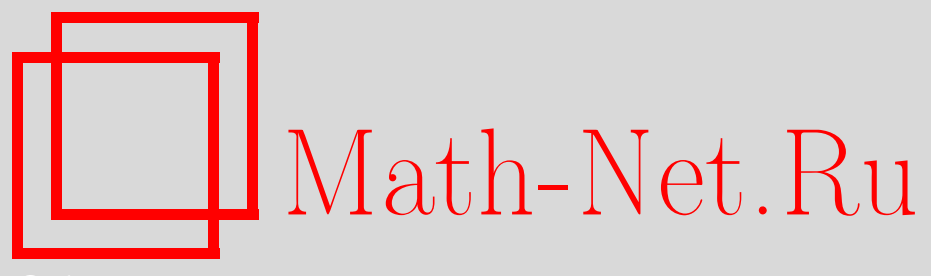

Ф. М. Мухамедов, О хаотическом поведении кубической $p$-адической динамической системы, Матем. заметки, 2008, том 83, выпуск 3, 468-471

DOI: https://doi.org/10.4213/mzm4531

Использование Общероссийского математического портала Math-Net.Ru подразумевает, что вы прочитали и согласны с пользовательским соглашением http://www . mathnet.ru/rus/agreement

Параметры загрузки:

IP : 54.147 .182 .235

26 апреля 2023 г., 18:06:11

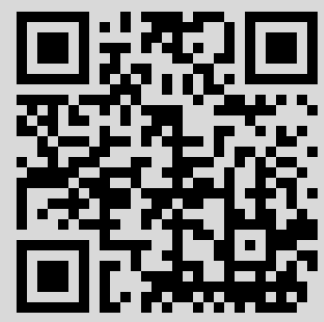




\section{О хаотическом поведении кубической $p$-адической динамической системы}

\section{Ф. Мухамедов}

Теория $p$-адических динамических систем развивалась главным образом в течение последних 15 лет [1], [2]. Неархимедова динамика в окрестности периодической и почти периодической точек над глобальными полями с применением локальных полей также изучалась в работах [3], [4]. В работе [5] была сформулирована задача получения полного описания аттракторов, т.е. дисков Зигеля для возмущаемых мономиальных динамических систем. В работе [2] изучались некоторые свойства возмущаемых динамических систем, задаваемых функциями $f_{q}(x)=x^{n}+q(x)$, где возмущение $q(x)$ является полиномом, коэффициенты которого имеют малые $p$-адические абсолютные значения. Была обнаружена связь между мономиальными и возмущаемыми мономиальными системами. Эти исследования показали, что изучение возмущаемых динамических систем имеет большое значение. Даже в случае квадратичной функции $f(x)=x^{2}+c, c \in \mathbb{Q}_{p}$, хаотическое поведение системы довольно сложное (см. [2], [6]). Поэтому, в данной короткой статье мы анонсируем некоторые результаты, касающиеся динамики $p$-адических кубических функций.

Напомним некоторые обозначения. Пусть $\mathbb{Q}$ - поле рациональных чисел. Здесь и далее, $p$ - фиксированное простое число. Любое рациональное число $x \neq 0$ можно представить в виде $x=p^{r}(n / m)$, где $r, n \in \mathbb{Z}, m$ - положительное целое число и $p, n, m$ - относительно простые числа; $p$-адическая норма числа $x$ задается выражением $|x|_{p}=p^{-r}$, $|0|_{p}=0$, и является неархимедовой, что означает, что она удовлетворяет неравенству ультраметрического треугольника $|x+y|_{p} \leqslant \max \left\{|x|_{p},|y|_{p}\right\}$. Пополнение поля $\mathbb{Q}$ относительно $p$-адической нормы определяет $p$-адическое поле, которое обозначается как $\mathbb{Q}_{p}$. Более детальное изложение теории $p$-адических полей можно найти в [7], [8].

Для любых $a \in \mathbb{Q}_{p}$ и $r>0$ будем писать

$$
\begin{gathered}
\bar{B}_{r}(a)=\left\{x \in \mathbb{Q}_{p}:|x-a|_{p} \leqslant r\right\}, \quad B_{r}(a)=\left\{x \in \mathbb{Q}_{p}:|x-a|_{p}<r\right\}, \\
S_{r}(a)=\left\{x \in \mathbb{Q}_{p}:|x-a|_{p}=r\right\} .
\end{gathered}
$$

Напомним некоторую стандартную терминологию теории динамических систем (см., например, [2]). Пусть $f: B \rightarrow B$ - аналитическая функция такая, что $B=B_{r}(a)$ или $\mathbb{Q}_{p}$. Введем обозначение $x^{(n)}=f^{n}\left(x^{(0)}\right)$, где $x^{0} \in B$ и

$$
f^{n}(x)=\underbrace{f \circ \cdots \circ f}_{n}(x) .
$$

Если $f\left(x^{(0)}\right)=x^{(0)}$, то $x^{(0)}$ называется неподвижной точкой. Неподвижная точка $x^{(0)}$ называется аттрактором, если существует такая окрестность $U\left(x^{(0)}\right)$ точки $x^{(0)}$, что для всех точек $y \in U\left(x^{(0)}\right)$ выполняется равенство $\lim _{n \rightarrow \infty} y^{(n)}=x^{(0)}$, где $y^{(n)}=f^{n}(y)$. Если $x^{(0)}$ является аттрактором, то его бассейном притяжсения является область

$$
A\left(x^{(0)}\right)=\left\{y \in \mathbb{Q}_{p}: y^{(n)} \rightarrow x^{(0)}, n \rightarrow \infty\right\} .
$$

Неподвижная точка $x^{(0)}$ называется отталкивающей точкой, если существует такая окрестность $U\left(x^{(0)}\right)$ точки $x^{(0)}$, что $\left|f(x)-x^{(0)}\right|_{p}>\left|x-x^{(0)}\right|_{p}$ для $x \in U\left(x^{(0)}\right), x \neq x^{(0)}$. Для неподвижной точки $x^{(0)}$ функции $f(x)$ шар $B_{r}\left(x^{(0)}\right)$ (содержащийся в $B$ ) называется

Работа выполнена при поддержке фонда FCT (грант SFRH/BPD/17419/2004). 
диском Зигеля, если любая сфера $S_{\rho}\left(x^{(0)}\right), \rho<r$, является инвариантной сферой функции $f(x)$, т.е., если точка $x \in S_{\rho}\left(x^{(0)}\right)$, то все ее итерации $x^{(n)}$ принадлежат $S_{\rho}\left(x^{(0)}\right)$ при всех $n=1,2 \ldots$. Объединение всех дисков Зигеля с центром в точке $x^{(0)}$ называется максимальным диском Зигеля и обозначается через $S I\left(x^{(0)}\right)$.

Пусть $x^{(0)}$ - неподвижная точка аналитической функции $f(x)$. Положим

$$
\lambda=\frac{d}{d x} f\left(x^{(0)}\right) .
$$

Точка $x^{(0)}$ называется притягивающей, если $0 \leqslant|\lambda|_{p}<1$, индифферентной, если $|\lambda|_{p}=1$, и отталкивающей, если $|\lambda|_{p}>1$.

В настоящей статье рассматривается динамическая система, соответствующая функции $f: \mathbb{Q}_{p} \rightarrow \mathbb{Q}_{p}$, определенной соотношением

$$
f(x)=x^{3}+a x^{2}, \quad a \in \mathbb{Q}_{p} .
$$

Непосредственная проверка показывает, что функция (1) имеет следующие неподвижные точки:

$$
x_{1}=0 \quad \text { и } \quad x_{2,3}=\frac{-a \pm \sqrt{a^{2}+4}}{2} .
$$

Заметим, что эти точки формально считаются неподвижными точками, поскольку, на самом деле, квадратный корень не всегда существует в $\mathbb{Q}_{p}$ и, следовательно, для полного исследования поведения динамики этой функции требуется существование неподвижных точек $x_{2,3}$.

Легко проверить, что неподвижная точка $x_{1}$ является притягивающей. Поэтому, в дальнейшем, будем рассматривать точки $x_{2,3}$. Следующие утверждения дают ответ на вопрос о существовании неподвижных точек в $\mathbb{Q}_{p}$ и об их поведении.

ПрЕДЛОЖЕНИЕ 1. Имеют место следующие утверждения.

(i) Пусть $|a|_{p}<1 ;$ тогда $\sqrt{a^{2}+4}$ существует в $\mathbb{Q}_{p}$ тогда и только тогда, когда либо $p \geqslant 3$, либо $p=2 u|a|_{p} \leqslant 1 / p^{3}$. В этом случае обе неподвижные точки $x_{2,3}$ являются притягивающими.

(ii) Пусть $|a|_{p}>1 ;$ тогда $\sqrt{a^{2}+4}$ существует в $\mathbb{Q}_{p}$. В этом случае неподвижная точка $x_{2}$ является отталкивающей, а точка $x_{3}$ - индифферентной.

(iii) Пусть $|a|_{p}=1 ;$ тогда $\sqrt{a^{2}+4}$ не существует в $\mathbb{Q}_{p}$ при $p=2$ или $p=3$. Если $p \geqslant 5 u\left|a^{2}+4\right|_{p}=1$, то $\sqrt{a^{2}+4}$ существует в $\mathbb{Q}_{p}$ тогда и только тогда, когда включение

$$
\frac{x^{2}-a_{0}^{2}-4}{p} \in \mathbb{Z}
$$

выполняется для некоторого $x \in \mathbb{Z}$. Если $\left|a^{2}+4\right|_{p}<1 u$

$$
\left\{\begin{array}{l}
a_{0}^{2}+4 \equiv 0(\bmod p), \\
\frac{a_{0}^{2}+4}{p}+2 a_{0} a_{1} \equiv 0(\bmod p), \\
a_{1}^{2}+2 a_{0} a_{2} \not \equiv 0(\bmod p),
\end{array}\right.
$$

то $\sqrt{a^{2}+4}$ существует. Здесь

$$
a=a_{0}+a_{1} p+a_{2} p^{2}+\cdots, \quad a_{0} \neq 0 .
$$

В этом случае либо обе неподвижные точки индифферентны, либо одна из них индифферентна, а другая является притягивающей точкой. 
Обозначим $r_{k}=1 /|a|_{p}^{k}, k \geqslant 1$. Для заданных множеств $A, B \subset \mathbb{Q}_{p}$ положим

$$
\begin{gathered}
T_{f, A, B}(x)=\min \left\{k \in \mathbb{N}: f^{(k)}(x) \in B\right\}, \quad x \in A, \\
D[A, B]=\left\{x \in A: T_{f, A, B}(x)<\infty\right\} .
\end{gathered}
$$

Теорема 2. Пусть $|a|_{p}>1$ и выполняется условие

$$
\sqrt{|a|_{p}} \notin\left\{p^{k}, k \in \mathbb{N}\right\} .
$$

Тогда для притягивающей неподвижной точки $x_{1}$ выполняется

$$
A\left(x_{1}\right)=B_{r_{1}}(0) \cup D\left[S_{1}(0) \cup S_{|a|_{p}}(0), B_{r_{3}}(-a)\right],
$$

а для индифферентной неподвижной точки $x_{3}$ выполняется

$$
\left|x_{3}\right|_{p}=r_{1} \quad u \quad S I\left(x_{3}\right)=B_{r_{1}}\left(x_{3}\right) .
$$

ЗАмечАниЕ 1. Заметим, что из сформулированной выше теоремы видно, что структура аттрактора точки $x_{1}$ довольно сложная. Для квадратичных динамических систем такой тип структуры аттрактора пока не известен (см., например, [2; гл. 8, раздел 4]).

Теорема 3. Пусть $|a|_{p}<1$. Выполняются следующие утверждения.

(i) Неподвижная точка $x_{1}$ является аттрактором и $A\left(x_{1}\right)=B_{1}(0)$.

(ii) Если $p \neq 3$, то неподвижные точки $x_{\sigma}, \sigma=2,3$, индифферентны и равенство $S I\left(x_{\sigma}\right)=B_{1}\left(x_{\sigma}\right)$ выполняется тогда и только тогда, когда $\sqrt{-3}$ существует в $\mathbb{Q}_{p}$. Иначе выполняется равенство $S I\left(x_{\sigma}\right)=\bar{B}_{1}\left(x_{\sigma}\right)$.

(iii) Eсли $p \geqslant 5$, mo $S I\left(x_{2}\right) \cap S I\left(x_{3}\right)=\varnothing$, а если $p=2$, mo $S I\left(x_{2}\right)=S I\left(x_{3}\right)$.

(iv) Если $p=3$, то неподвижные точки $x_{\sigma}, \sigma=2,3$ являются аттракторами и $A\left(x_{\sigma}\right)=B_{1}\left(x_{\sigma}\right)$.

ЗАмечАние 2. (2а) Заметим, что если динамическая система рассматривается над $p$-адическим комплексным полем $\mathbb{C}_{p}$, то получаемый результат отличается от сформулированной выше теоремы 3 , так как $\sqrt{-3}$ всегда существует в $\mathbb{C}_{p}$.

(2b) Стоит упомянуть, что результаты, аналогичные (i) и (iv), были получены в [2; гл. 4] при изучении возмущаемых свойств мономиальной динамики функции $x^{n}$. Оказалось, что подход теории возмущений не достаточно информативнен относительно геометрической структуры дисков Зигеля. Здесь и далее (см. теорему 4) проводится подробный анализ структуры этих дисков для рассматриваемой функции. Кроме того, результаты, аналогичные результатам, сформулированным в пунктах (ii)-(iii), просто не верны в случае квадратичных динамических систем.

Теорема 4. Пусть $|a|_{p}=1$. Выполняются следуюшие утверждения.

(i) Неподвижная точка $x_{1}$ является аттрактором и $A\left(x_{1}\right)=B_{1}(0) \cup D\left[S_{1}(0), B_{1}(-a)\right]$. Предположим, что неподвижные точки $x_{2}, x_{3}$ индифферентны.

(ii) Если $\sqrt{\left(a^{2}-6+a \sqrt{a^{2}+4}\right) / 2}$ существует в $\mathbb{Q}_{p}$, то $S I\left(x_{\sigma}\right)=B_{1}\left(x_{\sigma}\right)$. Иначе, $S I\left(x_{\sigma}\right)=\bar{B}_{1}\left(x_{\sigma}\right)$.

(iii) Пусть $\left|a^{2}+4\right|_{p}<1$. Если $\sqrt{-5}$ существует в $\mathbb{Q}_{p}$ nри $p>5$, mо $S I\left(x_{\sigma}\right)=B_{1}\left(x_{\sigma}\right)$. Иначе $S I\left(x_{\sigma}\right)=\bar{B}_{1}\left(x_{\sigma}\right)$. Кроме того, выполняется равенство $S I\left(x_{2}\right)=S I\left(x_{3}\right)$.

Предположим, что неподвижная точка $x_{2}$ - притягивающая.

(iv) $A\left(x_{2}\right)=B_{1}\left(x_{2}\right) \cup D\left[S_{1}\left(x_{2}\right), B_{1}\left(-2 x_{2}-a\right)\right]$. 
СледСтвие 5. Пусть $|a|_{p}=1,\left|a^{2}+4\right|=1, u x_{\sigma}-$ индифферентная неподвижная точка функции $f$. Если выполняется неравенство $\left|3 a^{2}-a+20\right|_{p}<1$, mо $S I\left(x_{\sigma}\right)=B_{1}\left(x_{\sigma}\right)$.

ЗАмечАние 3. (3а) При $|a|_{p}=1$ аналогичные виды динамических систем недавно изучались над комплексным р-адическим полем в работе Бенедетто [9]. Результаты, полученные в настоящей статье, отличаются от результатов, полученных там, поскольку мы пользовались свойством дискретности значений нормы $|\cdot|_{p}$ над $\mathbb{Q}_{p}$.

(3b) В основном, в случае, если возмущающий член мономиальной динамики нейтрален (т.е. $|q(x)|_{p}=1$ ), то изучение динамики становится очень трудным и метод теории возмущения перестает быть эффективным. Следовательно, если нейтральное возмущение присутствует в динамической системе, то следует изучать его природу. Например, динамическая система $f(x)=x^{2}+c$ при $|c|_{p}=1$ была полностью изучена в [2], и можно видеть, что структура аттракторов такой системы полностью отличается от нашей (см. также замечание $2,(2 \mathrm{~b}))$.

Исходя из полученных выше результатов и замечаний, приходим к выводу, что кубические динамические системы имеют более хаотическую структуру, чем квадратичные. Следовательно, задача, поставленная в [5] для кубических динамических систем, практически решена.

Автор выражает благодарность фонду FCT (Португалия) и анонимному рецензенту за полезные замечания.

\section{СПИСОК ЦИТИРОВАННОЙ ЛИТЕРАТУРЫ}

[1] A. Escassut, Analytic Elements in p-adic Analysis, World Sci. Publ., Singapore-New Jersey-London, 1995. [2] A. Yu. Khrennikov, M. Nilsson, p-adic Deterministic and Random Dynamics, Math. Appl., 574, Kluwer Acad. Publ., Dordreht, 2004. [3] J. Lubin, Compositio Math., 94:3 (1994), 321-346. [4] T. Pezda, Acta Arith., 66:1 (1994), 11-22. [5] С. Альбеверио, Б. Тироцци, А. Ю. Хренников, С. де Шмедт, ТМФ, 114:3 (1998), 349-365. [6] E. Thiran, D. Verstegen, J. Weyers, J. Statist. Phys., 54:3-4 (1989), 893-913. [7] N. Koblitz, p-adic Numbers, p-adic Analysis and Zeta-function, Grad. Texts in Math., 58, Springer-Verlag, Berlin, 1977. [8] A. M. Robert, A Course in p-adic Analysis, Grad. Texts in Math., 198, Springer-Verlag, New York, 2000. [9] R. Benedetto, C. R. Math. Acad. Sci. Paris, 335:7 (2002), 615-620.

\section{Ф. Мухамедов}

Поступило

Universidade de Aveiro, Португалия

03.05.2006

E-mail: far75m@yandex.ru, farruh@fis.ua.pt 